$$
\begin{aligned}
& \text { مقارنة لبعض طرائق تخمين التبخر - نتح المرجعي لمنطقة الموصل } \\
& \text { الآء عبد الله يعقوب } \\
& \text { مدرس مساعد / المعهز التقني موصل } \\
& \text { الخلاصة }
\end{aligned}
$$

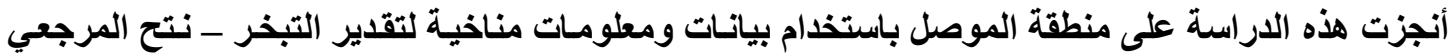

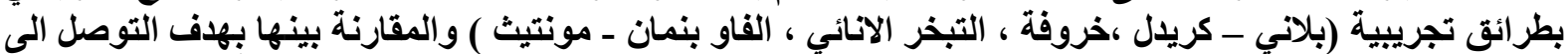

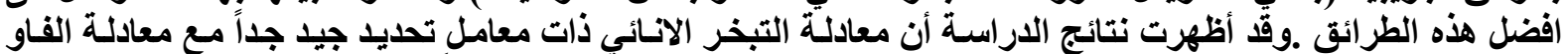

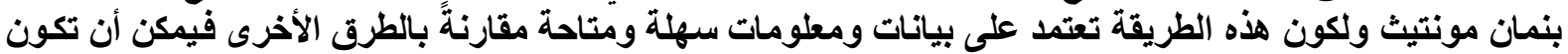

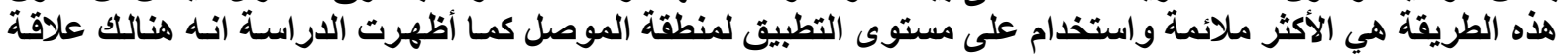

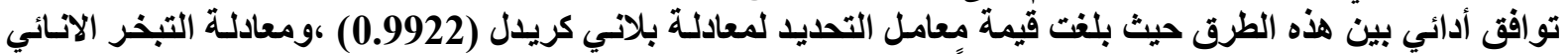

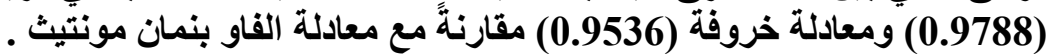

الكلمات الدالة : طرائق تخمين التبخر - نتح المرجعي ، منطقة الموصل

\title{
Comparison of some Methods to Estimation Reference Evapotranspiration for Mosul Area
}

\section{A. A.Yaqoob}

Technical Institute/Mosul

\begin{abstract}
This study has been established for Mosul area by using climatic data and information for estimation of evapotranspiration by using methods (Blany-Criddle), kharrufa, pan evaporation,Fao penman montieth) and comparison between them to reach to the best method this study showed that pan evaporation equation has a very good determination factor with Fao-penman montieth because this method depend on simple and easily obtained information in comparison with other methods so that this method is a more suitable use for Mosul area also this study showed that there is a correlation performance relationship between these method the value of determination factor for Blany - Criddle is (0.9922) and for pan evaporation is $(0.9788)$ and Kharrofa equation (0.9536) in comparison with Fao-penman Montieth .
\end{abstract}




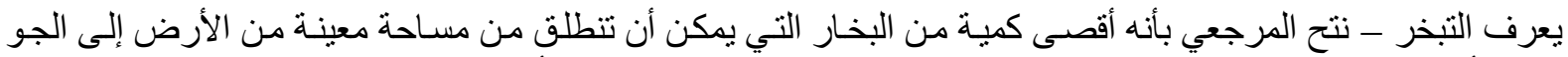

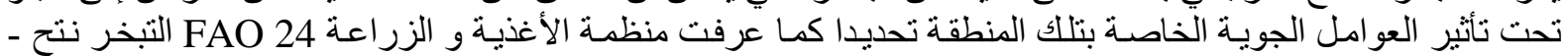

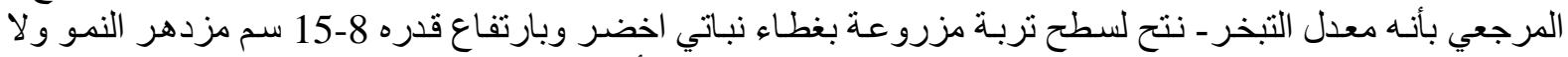

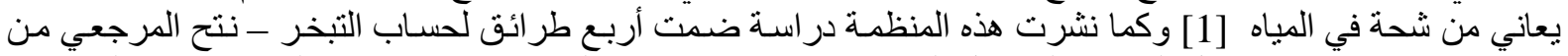

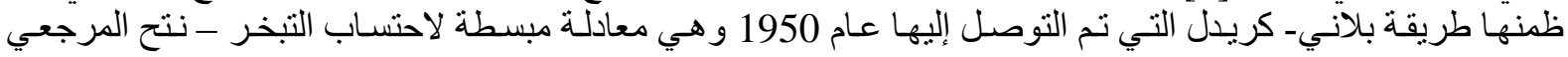

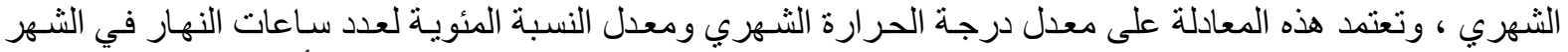

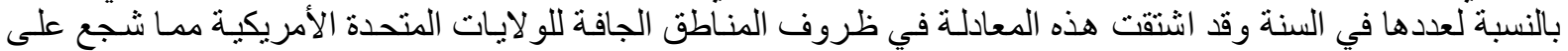

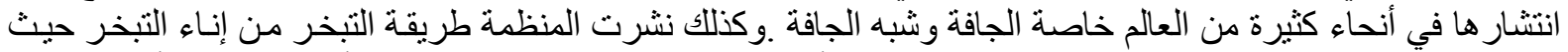

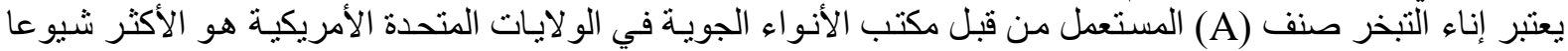

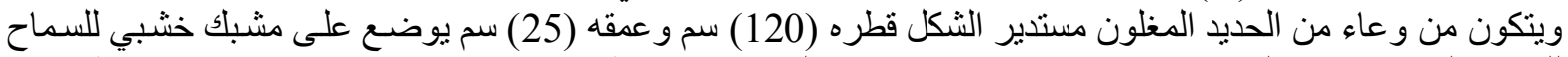

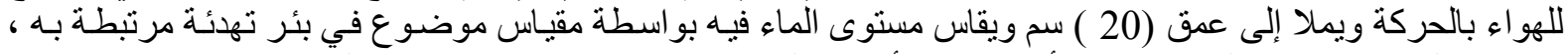

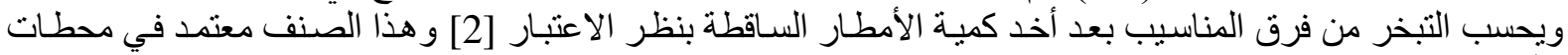

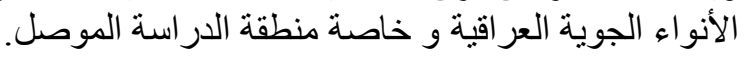

لقد توصل خروفة 1985إلى معادلة تجريبية بإجر اء تر ابط بين درجات الحر ارة بالمقياس المئوي و طول النهار من جهة

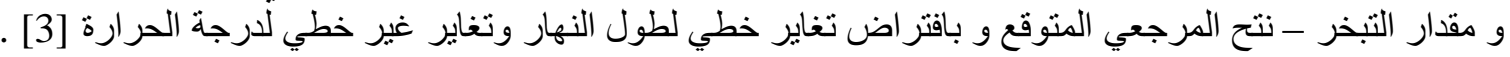

قامت منظمة الأغذية والزر اعة الدولية 1992 FAO بتكليف لجنة استثارية من الخبر اء الباحثين في مجال الري بتحليل

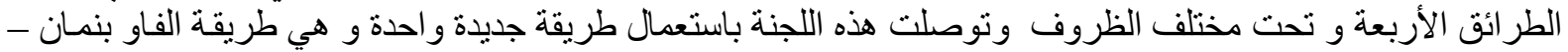

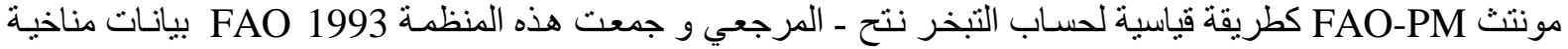

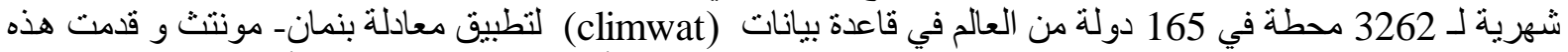

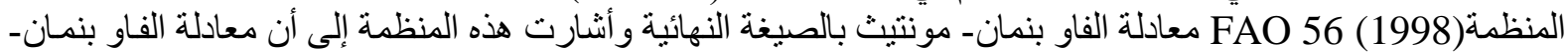

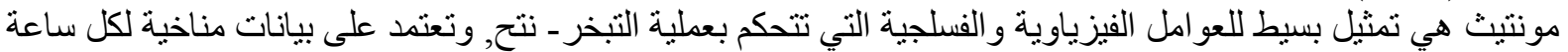

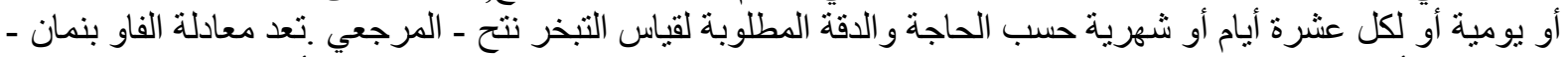

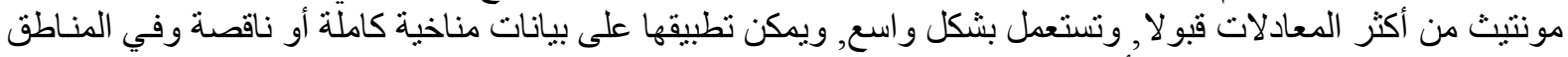

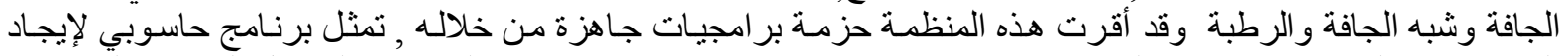

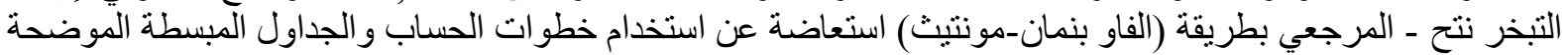
في بحث منظمة الأغذية والزر اعة المرقم - 56 - من بحوث الري والبزل [4) .

وطبقا لما سرد أعلاه كانت أهداف الدراسة الحالية هي تقدير التبخر - نتح المرجعي لمنطقة الموصل لأربع طر ائق هي (بلاني - كريدل , خروفه , التبخر الانائي ,الفاو بنمان - مونتيث) ومقارنة بين الطن الطر ائق .

\section{المواد وطرق البحث:}

1. اعتمدت البيانات المناخية اليومية للسنتين 2004, 2005 المأخوذة من المصدر (العاني و آخرون) [5] لمدينة الموصل

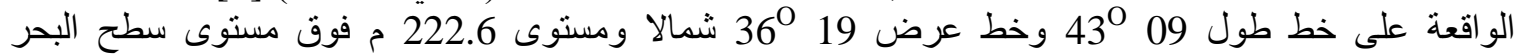

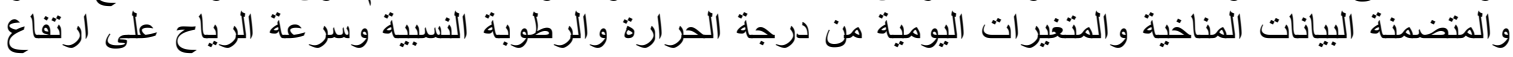

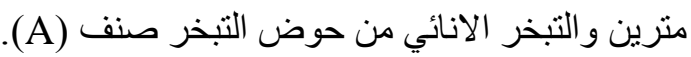

$E T p=P \cdot(0.46 \cdot T C+8.13)$

حيث أن

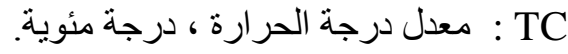

ETp P النسبة المئوية لعدد ساعات النهار في الثهر نسبة إلى عددها في السنة.

$$
\text { 3. تم حساب التبخر - نتح المرجعي بتطبيق معادلة (نجيب خروفة ) }
$$


C : : C

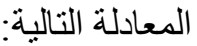

$$
C=0.22 \cdot\left(1+\frac{n}{N}\right) \cdot\left(0.9+\frac{W}{100}\right) \cdot(1-0.5 \cdot R H) \cdot\left(0.97+\frac{E}{10000}\right)
$$

= n

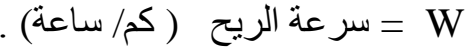
RH = RH = الرطوبة النسبية

4. تم حساب التبخر - نتح المرجعي بطريقة التبخر الانائي صنف (A) لمدينة الموصل باستخدام العلاقة:

$E T p=E p \cdot K p$

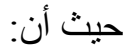

E = EP

(A) = K

و قد تم احتساب Kp لحوض اناه حوض التبخر صنف (A) باستخدام العلاقة :

$K p=0.108-0.0286 \cdot W+0.0422 \cdot \operatorname{In}(F)+0.1434 \cdot \operatorname{In}(R H)-0.00063 \cdot(\operatorname{In}(F))^{2} \cdot \operatorname{In}(R H)$

= سرعة الريح عند ارتفاع (2 مثر ) من سطح الأرض (مثر / ثانية).

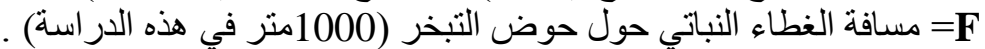

5. نم حساب التبخر - نتح المرجعي بطريقة الفاو بنمان - مونتيث باستخدام البرنامج الحاسوبي الجاهز ( CropWat)

[7, 6]: وحسب العلاقة التالية (version (4.3)

$E T p=\frac{0.408 \cdot \Delta \cdot(R n-G)+\gamma \cdot\left(\frac{900}{T+273}\right) \cdot W \cdot(e s-e d)}{\Delta+\gamma \cdot(1+0.34 \cdot w)}$

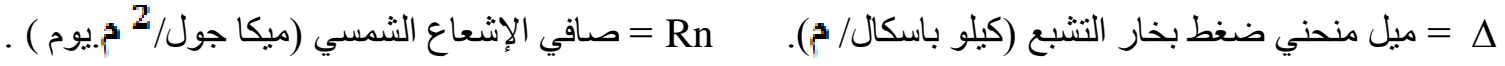
= G (es - ed)

\section{النتائج والمناقشة:}

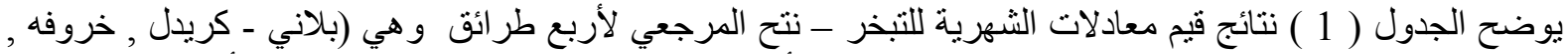

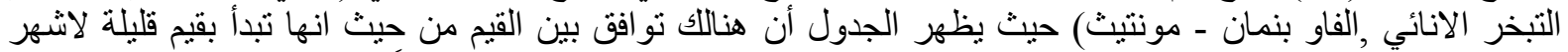

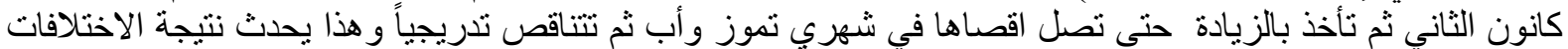

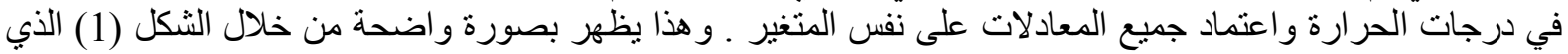

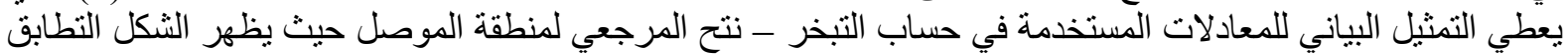

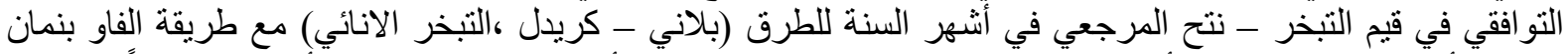

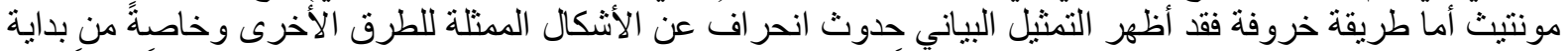

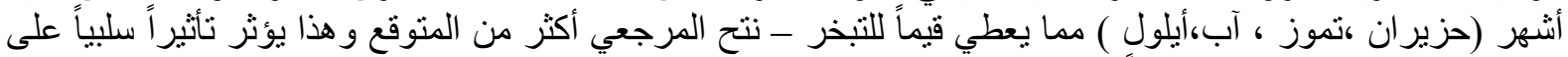


ولغرض تحديد التقارب مابين الطرق المستخدمة وطريقة الفاو بنمان مونتيث فقد تم رسم العلاقات الخطية الموضحة في

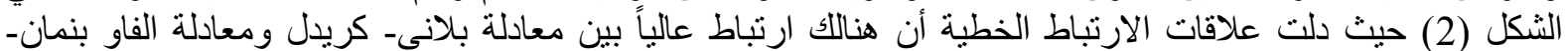

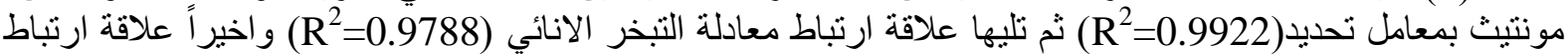
معادلة خروفة (R2=0.9563).

جدول(1) نتائج المعدلات الثهرية لقيم المعادلات المستخدمة لتخمين التبخر نتح المرجعي لسنوات الدراسة

\begin{tabular}{|c|c|c|c|c|c|c|c|c|}
\hline \multicolumn{8}{|c|}{ المعدلات الثهرية لقيم التبخر نتح المرجعي لسنوات الدراسة لمنطقة الموصل (ملم/يوم) } & \multirow{3}{*}{ 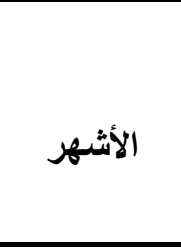 } \\
\hline \multicolumn{2}{|c|}{ الفاو بنمان_مونتيث } & \multicolumn{2}{|c|}{ التبخر الانائي } & \multicolumn{2}{|c|}{ 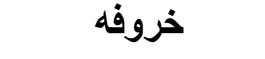 } & \multicolumn{2}{|c|}{ 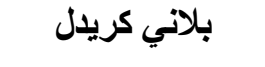 } & \\
\hline 2005 & 2004 & 2005 & 2004 & 2005 & 2004 & 2005 & 2004 & \\
\hline $\mathbf{0 . 7 8}$ & $\mathbf{0 . 8 3}$ & 1.01 & 1.06 & 1.12 & 1.41 & 2.65 & 2.81 & كانون ثاني \\
\hline 1.22 & 1.26 & 1.502 & 1.02 & 1.33 & 1.53 & 2.87 & 3.05 & 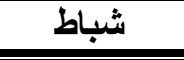 \\
\hline 2.38 & 2.32 & 2.31 & 2.75 & 2.73 & 3.12 & 3.88 & 4.06 & آذار \\
\hline 3.34 & 3.33 & 3.72 & 3.43 & 4.83 & 4.32 & 5.07 & 4.8 & نيسان \\
\hline 3.16 & 4.76 & 5.45 & 5.74 & 6.53 & 6.46 & 5.83 & 5.7 & أيار \\
\hline 6.16 & 6.15 & 7.75 & 7.7 & 7.13 & 9.51 & 7.22 & 7.3 & حزيران \\
\hline 6.61 & 6.44 & 7.88 & 8.38 & 10.89 & 10.85 & 7.79 & 7.7 & تموز \\
\hline 5.89 & 5.89 & 7.4 & 7.85 & 10.25 & 9.75 & 7.26 & 7.08 & آب \\
\hline 4.23 & 4.22 & 5.99 & 6.09 & $\overline{7.85}$ & 7.64 & 5.97 & $\overline{6.02}$ & أيلول \\
\hline 2.74 & 2.85 & 3.8 & 3.98 & 4.87 & 5.44 & 4.65 & 4.89 & تشرين الأول \\
\hline 1.43 & 1.43 & 1.68 & 1.26 & 2.49 & 2.51 & 3.39 & 3.4 & تشرين الثاني \\
\hline 0.7 & 0.68 & 1.24 & 0.88 & 1.86 & 0.98 & 2.98 & 2.51 & كاتون الأول \\
\hline
\end{tabular}

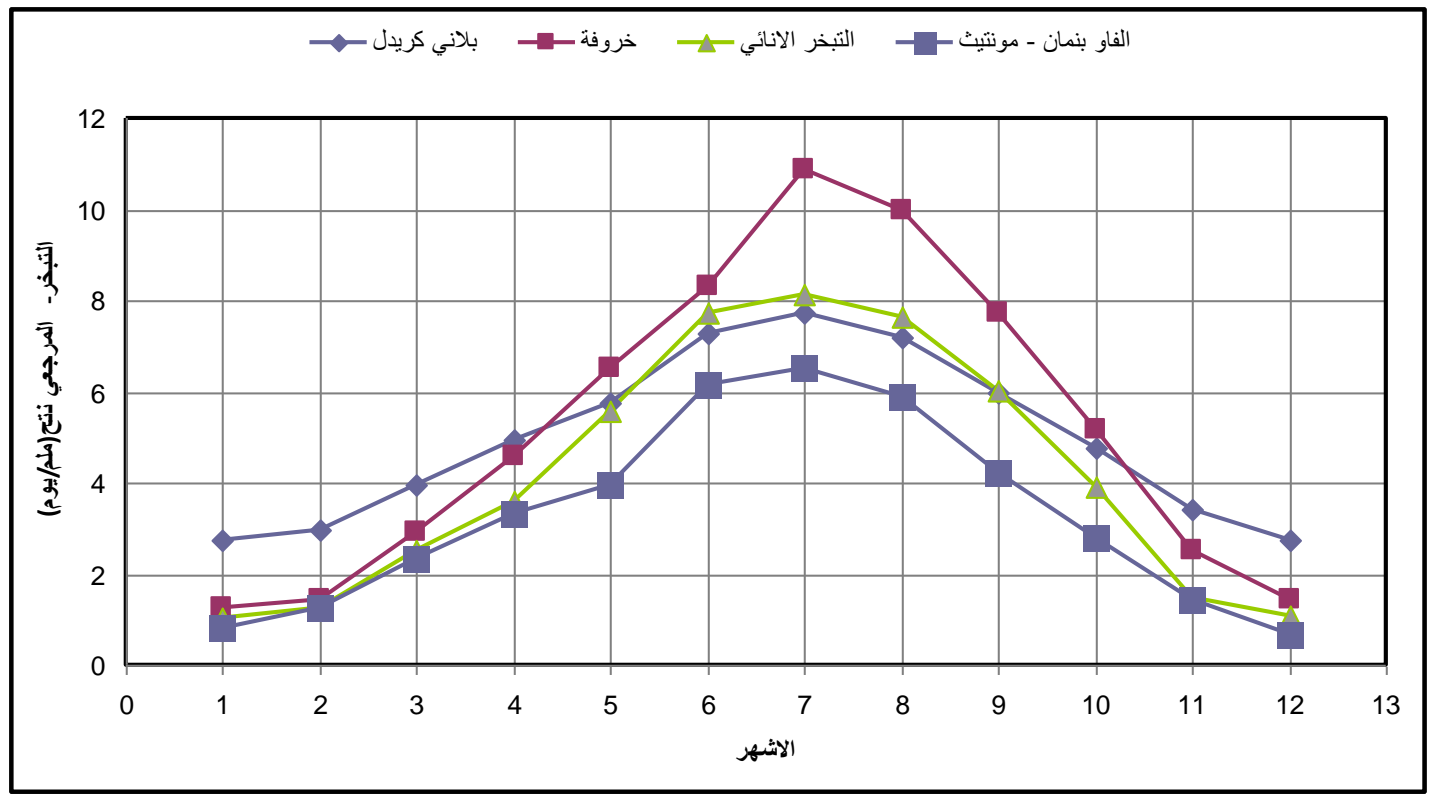

الثكل (1) المعدلات الثهرية للتبخر - نتح المرجعي المحسوب بالطرق الأربعة 
يعقوب : مقارنة لبعض طرائق تخمين التبخر - نتح المرجعي لمنطقة الموصل

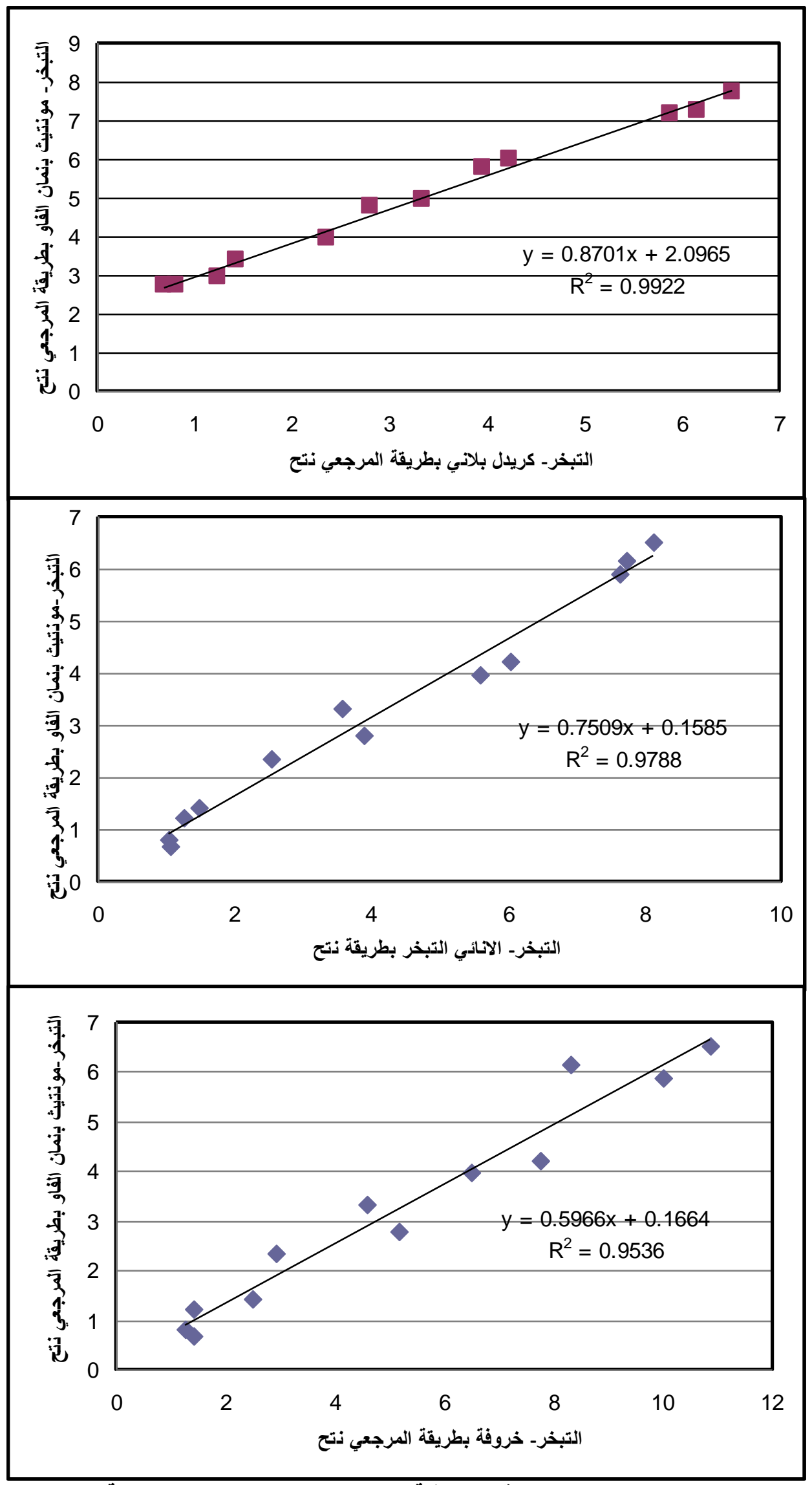

الثكل (2) العلاقات الخطية ومعاملات التحديد لمعادلات الاراسة 


\section{الاستتتاجات و التوصيات:}

من خلال النتائج يمكننا إدر اج الاستنتاجات التالية

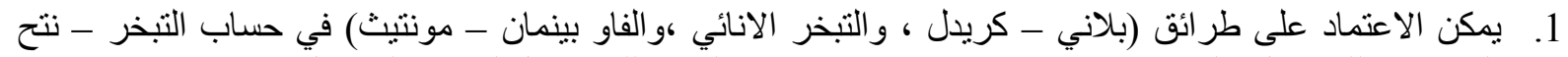

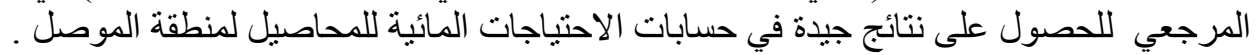

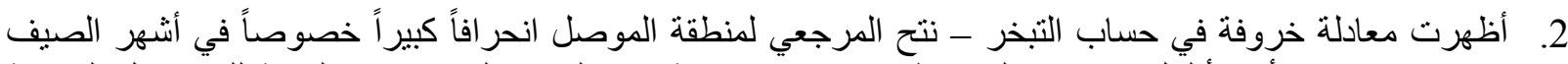
(حزير ان ،تموز ،أب ،أبلول ) مما يجعلها نعطي تقديرات تخمينية فوق المتوقع للاحتياجات المائية للمحاصيل الصيفية الصية المئية وبذللك بنصح بعدم استخدامها في المنطقة .

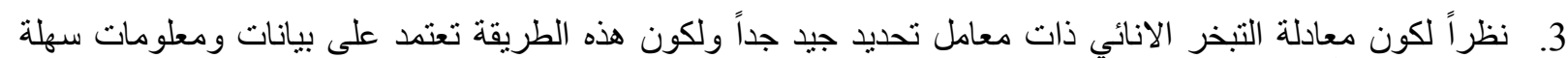

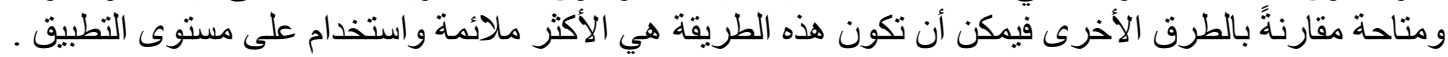

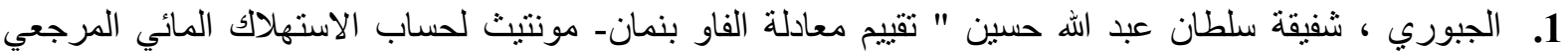

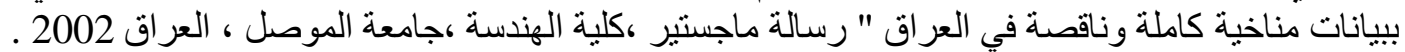

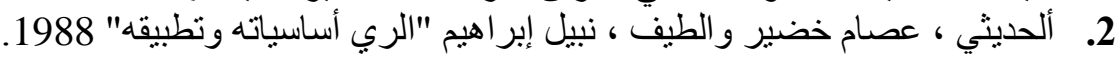

3. Kharrufa, N.S. 1985. Simplified equation for evapotranspiration in arid regions. Beitrage Zur Hydrologic. 5: 39-47.

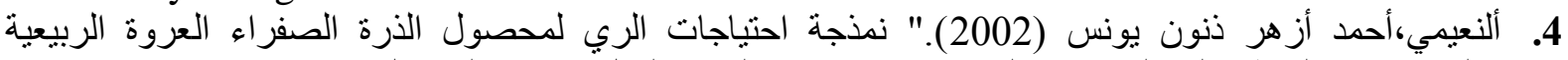

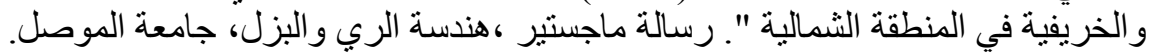

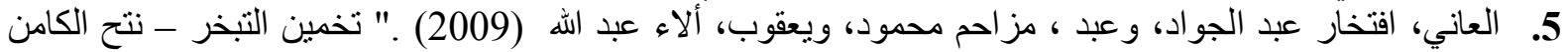

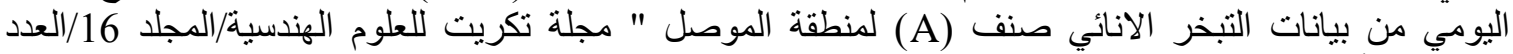

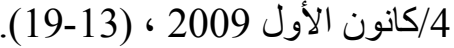

6. Allen R.G.pereira L.S , Raes, D., and smith, M., "Crop Evapotranpiration: Guidelines for Computing Crop Water Requirement.", Irrigation and Drainage paper, No. 56, Food and Agriculture Organization of the United Nations (FAO), R0me, 1998

7. Clark, D. (1998) " cropwat for windows user Guide, university of Southampton, version 4.2.oo13 October.

تم اجراء البحث في كلية ألهنسة = جامعة ألموصل 